Conclusions These results demonstrate that a high dose of Aspirin $300 \mathrm{mg}$ carries no significant increase in risk for developing IDA when given for a year post CABG.

\section{PTH-84 GI SAFETY PROFILE OF THE USE OF DOACS IN COMBINATION WITH ANTIPLATELETS IN CARDIOLOGY PATIENTS}

1,2Sonia Moteea* 1,2 Jessica Morrison, 1,2 Carol Firth, 1,2Sarah Tinker, 1,2Sulleman Moreea. ${ }^{1}$ Bradford Teaching Hospital, Bradford, UK; ${ }^{2}$ Bradford Teaching Hospitals Foundation Trust, Bradford BD9 6RJ, UK

\subsection{6/gutjnl-2021-BSG.263}

Background DOACs, such as apixaban (Ap), rivaroxaban (R), edoxaban (E) and dabigatran (D), are increasingly used instead of warfarin in atrial fibrillation, acute coronary syndrome and the prophylaxis/treatment of venous thromboembolism. Unlike warfarin, DOACs are used at a fixed dose and do not require close monitoring but the pivotal trials have shown an increased risk of GIB as compared to warfarin ${ }^{1}$. A recent meta analysis showed that the risk of GIB events related to DOACs (except Rivaroxaban) is not significantly greater than with warfarin ${ }^{2}$. There is therefore a need for more real world data.

Aims To review the real world safety profile of DOACs in combination with antiplatelets in patients who have been admitted to the cardiology wards.

Method For the period Jan 2015-Dec 2017 (36 months), we extracted the following data for all patients admitted under the cardiology team from our electronic databases: patient demographics; medication on discharge; patients having a gastroscopy (OGD); indication, finding and outcome at endoscopy.

Result During the study period, 4871 patients were admitted with a diagnosis of acute coronary syndrome (ACS). 729 (15\%) patients (456 M, mean age 62yrs; $273 \mathrm{~F}$, mean age $66 \mathrm{yrs})$ died during their admission, 2 of whom had an OGD for GIB prior to death but neither was on a DOAC. The remaining 4142 patients $(2677 \mathrm{M}$, mean age 63.8yrs; $1465 \mathrm{~F}$, mean age 67.5yrs) were on the following at discharge: monotherapy Aspirin (A) 1763, Clopidogrel (C) 555, Ticagrelor (T) 1095, R 83, Ap 12, E 3; dual therapy A+C/T/DOAC 1573; triple therapy $(\mathrm{A}+\mathrm{C} / \mathrm{T}+\mathrm{DOAC}) 69$

Clopidogrel + (Aspirin/DOAC/Ticagelor)- 508

Ticagrelor + (Aspirin/DOAC/Clopidogrel)- 1089

There were 449 gastroscopies (11\%) done during the study period and for 6 months thereafter. The indications were: GIB - 68 (15\%) (46M mean age 62, 23F, mean age 66), anaemia 215 (48\%), dyspepsia 157 (35\%).

Out of 68 patients with suspected GIB, there were 3 cases of active bleeding at the time of the OGD - X1 DU (on A), $\mathrm{X} 1$ Mallory Weiss tear, X1 duodenitis (both on A+T). There was 1 oesophagitis without active bleeding and the remaining 64 OGDs did not show any abnormality. There were no cases of acute GIB in patients on DOACs in this cohort.

Including the 2 patients who had a GIB and died (mortality $0.05 \%$ ), there were in total 5 cases of acute GIB at the time of OGD $(0.12 \%$ severe GIB risk).
Conclusion Allowing for the retrospective nature of the study, the short follow up for some patients and the lack of information on the concurrent use of PPIs, our real world study shows a very low GIB risk for cardiology patients on antiplatelets +/- DOACs $(0.12 \%)$. The mortality in this cardiology cohort was also very low. This compares well with the published 1\% risk for GIB for patients on DOACs for all other indications ${ }^{2}$. Our results are therefore very reassuring.

\section{REFERENCE}

1. Holster, et al. Gastroenterology. 2013;145:105-112.e15.

2. Gu, et al. CGH 2019. DOl: https://doi.org/10.1016/j.cgh.2019.05.056

\section{PTH-85 GASTRODUODENAL TUBERCULOSIS: A GLOBAL LITERATURE REVIEW AND MANAGEMENT ALGORITHM FOR CLINICAL DECISION MAKING}

Rukmini Jagdish*, Olivia Etter, Georgina Chadwick, Samuel Pannick, Helen Burgess, Moe Kyaw. Chelsea and Westminster Hospital NHS Foundation Trust, London, UK

\subsection{6/gutjnl-2021-BSG.264}

Introduction Gastroduodenal tuberculosis (GDTB) is a rare form of tuberculosis. Clinical decision making around management remains unclear and complex.

Our aim was to review case reports on GDTB patients and their outcomes following intervention and formulate an algorithm to support clinicians when managing GDTB patients.

Methods A broad literature search across multiple databases (PubMed, Web of Science, Science Direct, Google Scholar, Cochrane Library) was conducted with the following keywords: pyloric, gastric outflow obstruction, gastroduodenal and tuberculosis. Data on patient demographics, presentation, endoscopy findings, intervention and outcome were extracted from publications meeting the inclusion criteria. A positive outcome included: improvement in symptoms, weight gain or resolution at follow up endoscopy. Descriptive statistics were applied.

Results Our search identified 29 relevant papers compiled of data from 63 patients. The average age of patients was 35 years, with $56 \%(n=23)$ of cases being male. A positive outcome was seen in 26/28 patients (93\%) treated with AntiTuberculous Treatment (ATT) alone and 26/31 patients (84\%) managed surgically followed by ATT. The average time in months to resolution of symptoms was 6.67 months for ATT alone and 8 months for surgery followed by ATT. A higher proportion of those who had surgery followed by ATT were diagnosed with operative histology $(29 \%, \mathrm{n}=31)$ compared to those treated with ATT alone $(3.6 \%, n=28)$. These results were interpreted and a management algorithm was proposed. (Figure 1).

Conclusions This is the largest analysis of case reports reporting patient outcomes following intervention for GDTB worldwide. ATT alone has a significant role in management of GDTB across varied presentations and disease severity. We recognise the importance of the diagnostic method and its impact on the choice of management. The role of endoscopic intervention for GDTB requires further research. 